\title{
Virological surveillance of the Influenza A(H1N1)2009 pandemic: the role of the Belgian National Influenza Centre
}

\author{
by \\ Gérard $C^{1}$, Brochier $B^{1}$, Quoilin $S^{2}$, Wuillaume $F^{2}$, Van Casteren $V^{2}$, Thomas ${ }^{1}$
}

\section{Keywords}

Surveillance, Influenza, diagnosis, PCR

\section{Introduction}

On 24 April 2009, the World Health Organization (WHO) first reported the emergence of a new Influenza virus in the United States of America and Mexico (1). This novel Influenza virus was identified as a new subtype $A(H 1 N 1)$ resulting from a reassortment of avian, human and swine Influenza viruses. This virus, which was later on referred to as A(H1N1)2009, rapidly demonstrated its capacity to transmit among humans (2). On 11 June 2009, the WHO decided to raise the pandemic alert phase to its maximum level (phase 6) (3).

Influenza in humans is caused by one of three types of influenza viruses A, B and rarely C, which all belong to the orthomyxoviridae family, and have two glycoproteins on the surface of the virions, the hemagglutinin (HA) and the neuraminidase (NA). These proteins elicit antibody responses to the Influenza virus, and so far sixteen different HAs (H1 to H16) and 9 different NAs (N1 to N9) have been recognised. Several combinations of HA and NA proteins are possible, each combination representing a different subtype (for example $A / H 1 N 1$, $\mathrm{A} / \mathrm{H} 3 \mathrm{~N} 2, \mathrm{~A} / \mathrm{H} 1 \mathrm{~N} 2 \ldots)$. . An important characteristic of Influenza viruses is their ability to evolve continuously to escape the immune response. The mechanisms behind this evolution are either antigenic drift (point mutations in the HA and NA genes) or antigenic shift (reassortment between different Influenza viruses).

For a virus to cause a pandemic, two major criteria must be met: the virus must be novel (antigenic shift), as this means that a large proportion of the population is susceptible to infection, and the virus must be transmissible from person to person $(4,5)$. In this context, the initial reporting of this novel transmissible Influenza variant to the WHO and the continuous surveillance of this virus is of the highest importance and allows to monitor virus evolution and reduce the public health risk for the population worldwide (6).

\footnotetext{
Scientific Institute of Public Health, Operational Direction Transmitted and Infectious Diseases, Virology Unit, Brussels, Belgium

2 Scientific Institute of Public Health, Operational Direction Public Health and Surveillance, Brussels, Belgium carine.gerard@wiv-isp.be
} 
In Belgium, the surveillance is ensured by the National Centre for Influenza (NIC) (www.wivisp.be/flu). According to the WHO $(7,8)$, the responsibilities of the NIC during the pandemic period are: 1) to provide laboratory diagnosis for monitoring the geographical spread of the pandemic in the country; 2) as the pandemic intensifies and becomes widespread, to adjust virological surveillance in order to monitor the progress of the pandemic in the country; 3 ) to maintain adequate virological surveillance to assist WHO in monitoring for example antigenic and genetic changes in the pandemic virus, pathogenicity, and antiviral susceptibility.

\section{Methods}

Sampling in the containment phase

From 26 April to 13 July 2009, a containment phase was implemented according to the WHO and CDC recommendations, in order to limit the extension of the epidemic (9). The Interministerial Influenza Coordination Committee established a series of measures and protocols to manage all suspect cases and contacts regarding notification, sampling, prophylaxis, treatment and isolation (10). An active surveillance system was then established, in order to detect all suspect cases of $\mathrm{A}(\mathrm{H} 1 \mathrm{~N} 1) 2009$ and to trace their contacts. Physicians, in collaboration with the communities' health inspectors, were asked to take a sample from each patient showing flu symptoms and returning from infected countries or regions. Due to the rapid geographic extension of the pandemic, the list of such countries was updated regularly.

During this period, more than 3000 sampling kits were sent to physicians, laboratories and hospitals all around Belgium and additional kits were provided on request. These kits each contained 4 swabs, transport medium, FFP2 masks, an information form and a detailed sampling procedure to take respiratory secretions from nose and throat. Samples needed to be sent to the NIC on the same day, in order to give a diagnostic result within 5-6 hours. The laboratory was operational $24 / 24$ hours, $7 / 7$ days, in collaboration with all involved public health authorities.

Sampling in the mitigation phase

From 13 July 2009 onwards, because of the sustained community spread, Belgium's strategy shifted to mitigation (week 29 to recent), where no systematic laboratory confirmation testing was requested anymore. Only high risk and hospitalised patients were to be tested for $A(H 1 N 1) 2009$ if suspected.

In addition, during this phase, the NIC strengthened the nationwide surveillance system to study the $A(H 1 N 1) 2009$ virus circulation in the Belgian population and to help reduce influenza morbidity and mortality. Therefore the influenza virological sentinel surveillance network, previously composed of 98 general practitioners (GPs) distributed in the whole country (11), was expanded to 139 GPs (78 GPs located in Flanders, 49 in Wallonia and 12 in the Brussels-Capital Region) (Figure 1). These GPs were all part of the Sentinel GP Network composed of approximately $160 \mathrm{GPs}$, who are also involved in the clinical influenza 
surveillance (11). The decision to enlarge the virological sentinel surveillance network was taken when the first three positive $\mathrm{A}(\mathrm{H} 1 \mathrm{~N} 1) 2009$ cases in Belgium were diagnosed by this network during week 27.

Figure 1: Geographical distribution (N) by district of the GPs involved in the influenza virological sentinel surveillance in Belgium

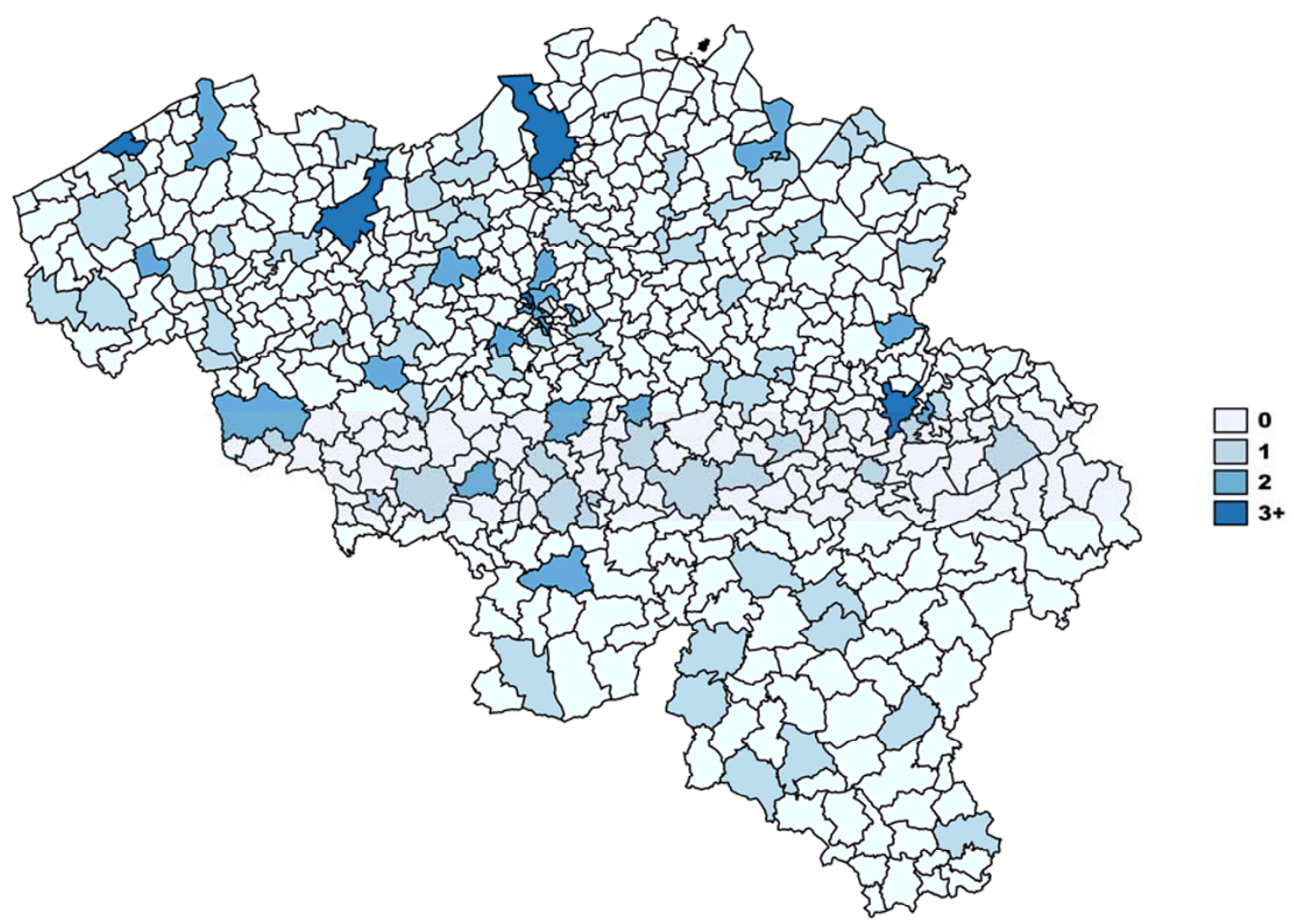

Laboratory testing

At the start of the pandemic, there was still a lack of information about the pathogenicity and transmission of the virus. Therefore, and to avoid any possible reassortment between strains from swine and human origins, manipulation and RNA extraction of the suspect specimens were performed in biosafety level 3 (BSL3) facilities instead of BSL2 where similar samples are tested yearly within the framework of monitoring seasonal flu. Viral RNA from clinical samples was extracted in duplicate with the QIAamp® Viral RNA Kit from QIAGEN (Hilden, Germany).

The extracted RNA was then submitted to a series of real-time PCR tests in a BSL2 environment. The real-time PCR reactions were performed in a Stratagene Mx3000P TM thermal cycler using the SuperScript ${ }^{\circledR}$ III Platinum ${ }^{\circledR}$ One-Step qRT-PCR System from Invitrogen (Carlsbad, California, USA). At first, there was no specific test available for the new pandemic influenza virus. Therefore the suspect samples were tested for influenza $A$ and $B$, and 
subtyped for seasonal influenza $A / H 1$ and $A / H 3$. Primers and probes for each type $(A / B)$ and subtype $(\mathrm{H} 1 / \mathrm{H} 3)$ were designed in a conserved gene region, selected either from the literature or after multiple-sequence alignment with a large amount of recent human and animal strains available at GenBank. Regions of the matrix gene (12) and of the Hemagglutinin gene (13) were amplified for typing $A$ and $B$ respectively. For subtyping, a region in the HA gene was amplified. A sample which was positive for influenza $A$ and negative for both seasonal subtypes ( $\mathrm{H} 1 \mathrm{~N} 1$ and $\mathrm{H} 3 \mathrm{~N} 2)$ was considered as a probable case.

From 3 May 2009, specific primers and probes for $A(H 1 N 1) 2009$ virus were sent by the Centers for Disease Control and Prevention (CDC) of Atlanta (14) and used as standard tests in our laboratory. The workflow of the tests is shown in Figure 2.

Figure 2: Workflow of laboratory tests in case of influenza $A(H 1 N 1) 2009$ suspicion in a patient

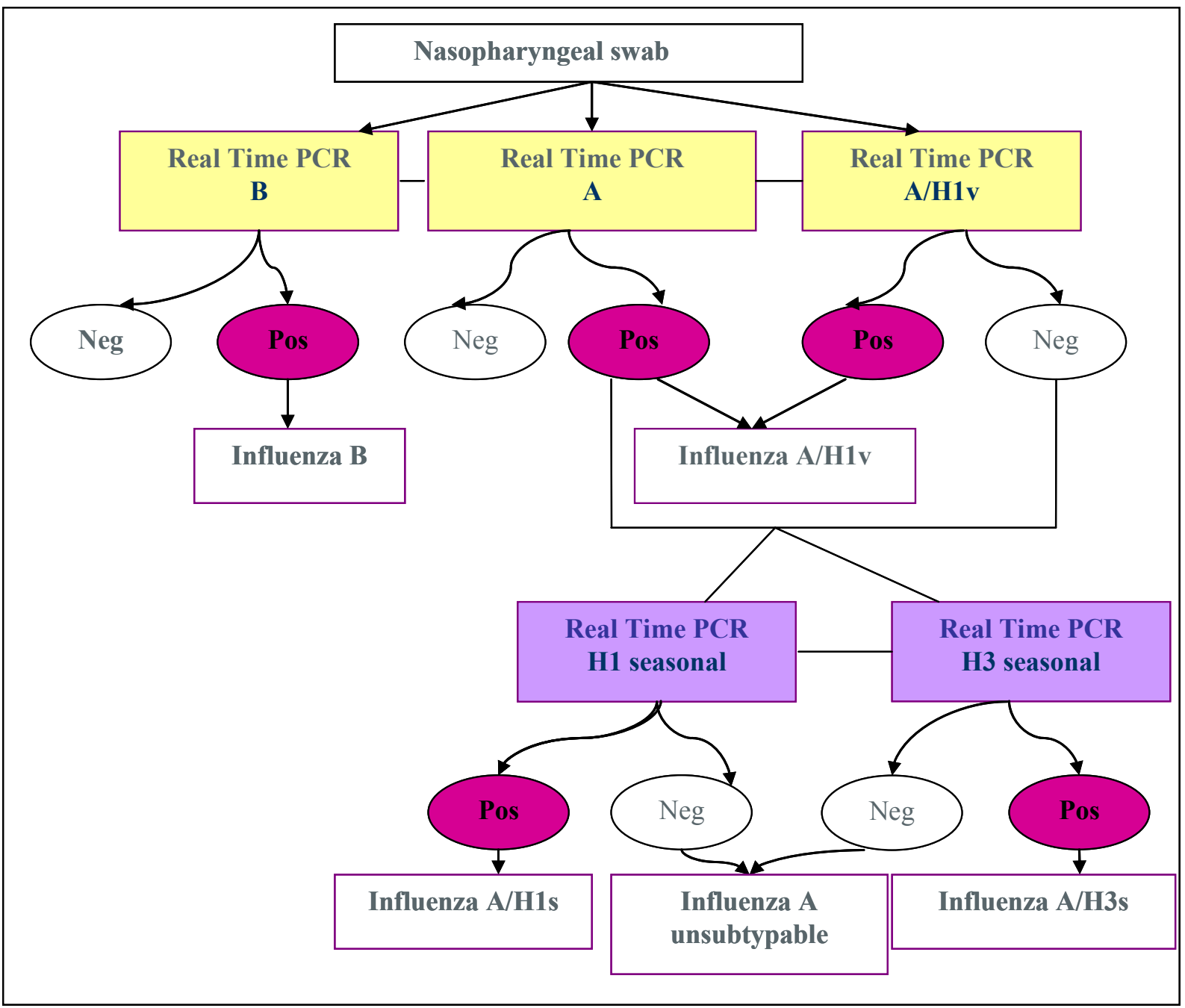

A series of randomly selected samples from the start, the peak and the end of the pandemic, as well as samples from more severely affected patients, were also sequenced, in order to detect any mutations in the hemagglutinin or neuraminidase gene, including mutations asso- 
ciated with resistance to neuraminidase inhibitors. A selection of approximately 200 samples was also sent to London (WHO Collaborating Centre) for antigenic characterisation and monitoring of antiviral resistance.

\section{Results}

On 12 May 2009, the NIC confirmed the first case of influenza $A(H 1 N 1) 2009$ in a patient returning from the United States. In total, 614 patient samples were tested during the containment phase (week 18 to 28) in Belgium, of which 125 cases were confirmed positive for $A(H 1 N 1) 2009$, the majority being imported cases. One sample out of the 614 analysed samples was positive for influenza $B, 14$ samples were confirmed to be $A / H 3 N 2$ positive and 13 others were found to be positive for influenza A but could not be subtyped. This could be due to the lower sensitivity of the PCR test for subtyping $\mathrm{H} 1$ in comparison with the PCR for typing $A$ or due to the evolution of the virus causing mismatches with the primers or probes.

During the mitigation phase, 729 additional suspect cases were tested, with 285 confirmed cases for $A(H 1 N 1) 2009,3$ cases positive for influenza $A / H 3 N 2$ and 47 cases positive for influenza A which could not be subtyped for similar reasons as mentioned above.

Figure 3: Weekly evolution of the number of analysed samples and influenza A positive samples from week 2009-18 until week 2009-52 in the non-sentinel network

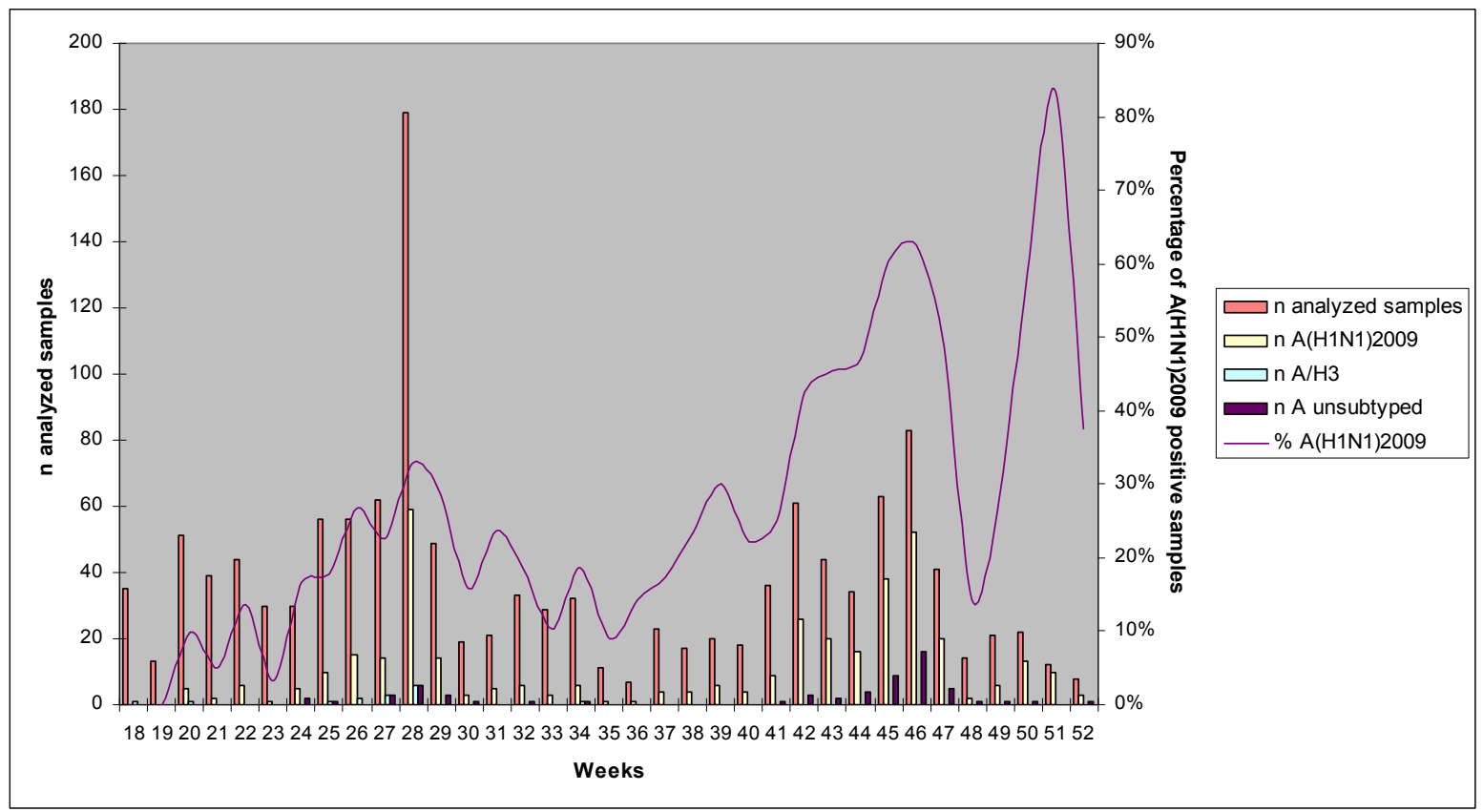

Figure 3 shows the weekly evolution of the number of analysed samples and the number of confirmed $\mathrm{A}(\mathrm{H} 1 \mathrm{~N} 1) 2009$ or $\mathrm{A} / \mathrm{H} 3 \mathrm{~N} 2$ cases in the non-sentinel network. In the first phase of the pandemic, a high number of suspected samples received from physicians, laboratories and hospitals all around Belgium was analysed but did not necessarily reveal a high number of $A(H 1 N 1) 2009$ confirmed cases in relation to the total number of analysed samples. This is especially true for week 28 . Several factors could be involved: the low incidence of Influenza 
$A(H 1 N 1) 2009$ at that time, the high media attention to the flu crisis, the subsequent psychological impact on the population and also the first people returning form holidays in countries where the novel influenza strain was already detected. A second peak in the number of analysed samples was observed in week 46 , which coincides more or less with the epidemic peak, characterised by a high percentage of confirmed $A(H 1 N 1) 2009$ cases. A low number of influenza $A / H 3 N 2$ could still be detected until week 34 , which might correspond to the end of the seasonal influenza epidemic 2008-2009 which was characterised by a high prevalence of the $A / H 3 N 2$ subtype. In week 21, an increase in the percentage of $A(H 1 N 1) 2009$ positive samples was observed, resulting from a higher number of confirmation tests (samples already tested positive for influenza $A$ but still not subtyped by other laboratories) at this stage of the epidemic.

Figure 4 shows the weekly evolution of the number of analysed samples and of influenza $\mathrm{A}(\mathrm{H} 1 \mathrm{~N} 1) 2009$ positive samples in the sentinel network. In Belgium, the proportion of positive samples increased from $28 \%$ in week 40 to $73 \%$ in week 44 (corresponding to the epidemic peak) (11). The proportion then decreased slowly from week $46(51 \%)$ to week $52(25 \%)$. From week 43 to week 44, the graph shows a drastic decrease in the number of analysed samples. This is the result of a change in the sampling strategy. Until that time, GPs were asked to take a sample from each patient who presented with influenza-like illness (ILI) symptoms. But from then on, they were asked to take maximum 2 samples a week, in order to decrease the number of analyses. Still, until week 50 , the epidemiological criteria were respected: the rate of doctor visits was still above the epidemic threshold (established at 141 doctor visits per 100,000 inhabitants) and among patients with ILIs, more than $20 \%$ people were found positive for influenza A(H1N1)2009 (Figure 4).

Figure 4: Weekly evolution of the number of analysed samples and influenza A positive samples from week 2009-19 until week 2009-52 in the virological sentinel network

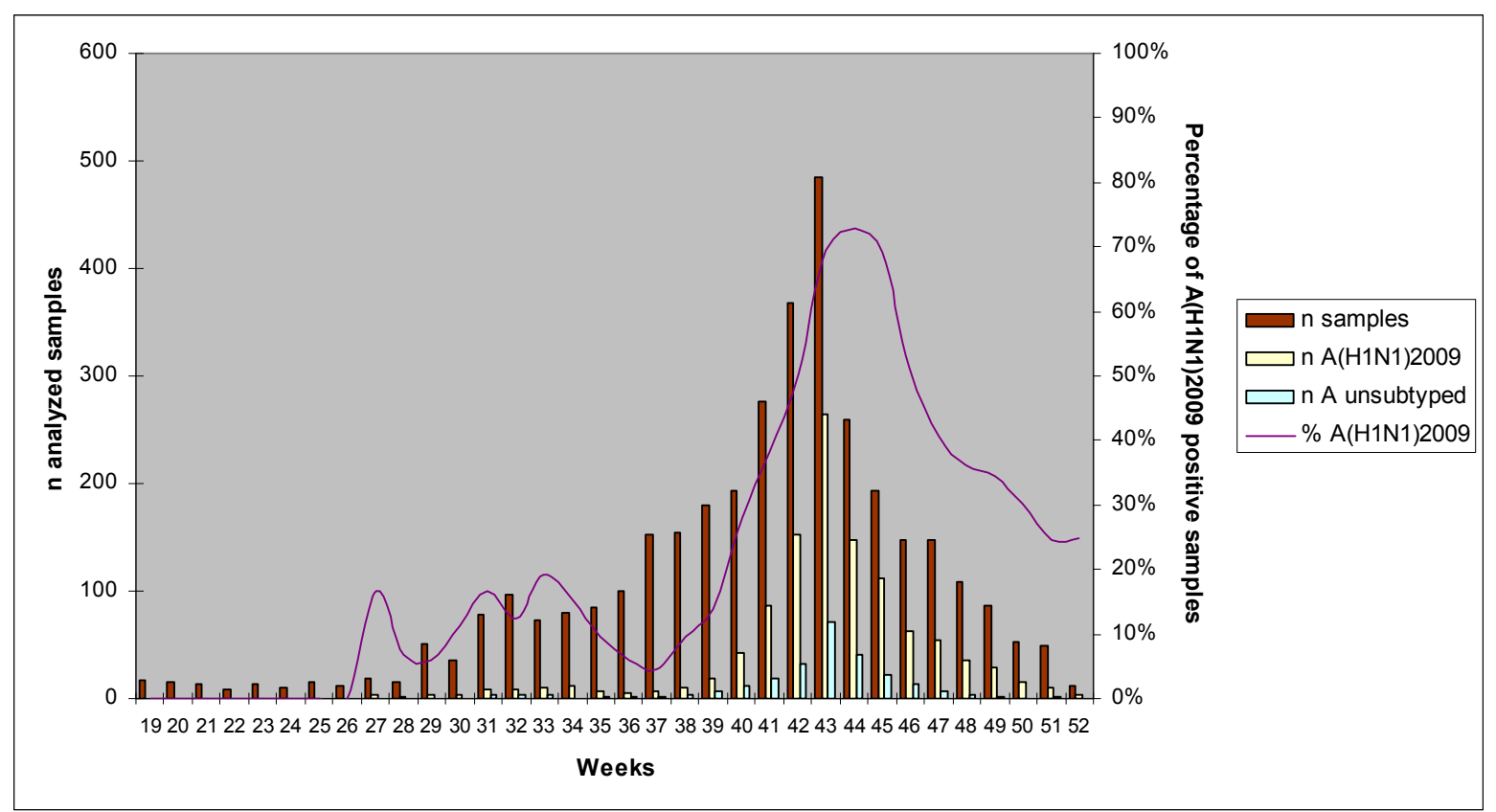


The genetic and antigenic analysis of a series of $\mathrm{A}(\mathrm{H} 1 \mathrm{~N} 1) 2009$ strains isolated in Belgium revealed no significant difference with pandemic strains found worldwide. All strains correspond to the reference strain A/California/4/09. Out of the 48 samples analysed by the WHO Collaborating Centre, one strain isolated from an immunosuppressed patient showed resistance to Oseltamivir (neuraminidase inhibitor). In this patient, the mutation H275Y was observed in the neuraminidase gene.

\section{Conclusions}

The introduction of the Influenza A(H1N1)2009 virus in Belgium occurred in a similar manner as in other European countries, initially causing a small but rapidly increasing number of cases (10). In this emergency context, the laboratory optimised a battery of real-time RTPCR assays to be able to perform diagnosis within one day. The aim was to identify the positive cases as soon as possible and to limit the propagation of the virus in the Belgian population. The surveillance system worked in accordance with the NIC public health mission, demonstrating an effective collaboration between the different partners and national authorities. Until the end of 2009 , the pandemic appeared to be less severe than initially predicted, with less serious symptoms and a limited number of lethal cases. Finally its evolution seems to be more comparable with that of a classical seasonal epidemic. Genotypically, the pandemic influenza strains isolated in Belgium up to now correspond to those isolated worldwide.

Nevertheless, future evolution of the $A(H 1 N 1) 2009$ virus remains uncertain, even if this pandemic virus will probably circulate for the next seasons. Continuous efforts must be made to update the molecular diagnostic methods to follow the high genetic variability of influenza viruses and to highlight potential resistance against antivirals. Therefore a close and sustained monitoring of the evolution of the virus is required to provide an adequate response and surveillance system in the near future.

\section{Acknowledgements}

This surveillance is financially supported by the Directorate-General for Primary Health Care and Crisis Management (DG2) of the Federal Public Service Health, Food Chain Safety and Environment and by the French and Flemish Communities.

Many thanks also to Christophe Van den Poel, Ilham Fdillate, Mona Abady, Regine De Bruyn and Yvonne Ronflette for their intensive work and their constant enthusiasm. A special thanks to Anneleen Hombrouck for reviewing the article and Jérôme Antoine for designing the figures. And finally, thanks to Totje, IMleke, my friends and my so understanding family for always being there. 


\section{References}

1. Centers for Disease Control and Prevention (DCD). Swine Influenza A (H1N1) infection in two children Southern California, March-April 2009. MMWR Morb Mortal Wkly Rep 2009; 58(15):400-2

2. Novel Swine-Origin Influenza A (H1N1) Virus Investigation Team. Emergence of a Novel Swine-Origin Influenza A (H1N1) Virus in Humans. N Engl J Med 2009; 360:2605-15

3. World Health Organization. World now at the start of 2009 influenza pandemic. Statement to the press by WHO Director-General Dr Margaret Chan. 11 June 2009. Available from: http://www.who.int/mediacentre/news/statements/2009/h1n1_pandemic_phase6_20090611/en/index.html

4. The Writing Committee of the WHO Consultation on Human Influenza A/H5. Avian Influenza A (H5N1) Infection in Humans. N Engl J Med 2005; 353:1374-85

5. Poland GA, Jacobson RM, Targonski PV. Avian and pandemic influenza: An overview. Vaccine 2007; 25: $3057-61$

6. WHO Case definition for human infections with influenza A (H5N1) virus. Geneva, World Health Organization, 2006

7. The role of National Influenza Centers (NICs) during Interpandemic, Pandemic Alert and Pandemic Periods. Interim Document, 2007. Available from:

http://www.who.int/csr/disease/avian_influenza/guidelines/RoleNICsMayf.pdf

8. Guidance to Influenza Laboratories Diagnosing Swine Influenza A(H1N1) Infections of Current Concern. 25 April 2009. Available from: http://www.who.int/csr/disease/swineflu/swineflu_guidance_labs_20090425.pdf

9. World Health Organization (WHO). Swine Influenza. Statement to the press by WHO Director-General Dr Margaret Chan. 25 April 2009. Available from: http://www.who.int/mediacentre/news/statements/2009/h1n1_20090425/en/index.html

10. Belgian working group on influenza $A(H 1 N 1) v$. Influenza $A(H 1 N 1) v$ virus infections in Belgium, May-June 2009. Euro Surveill. 2009; 14(28):pii=19270

11. Van Casteren V, Mertens K, Antoine J, Wanyama S, Thomas I, Bossuyt N. Clinical surveillance of Influenza $A(H 1 N 1) 2009$ pandemic through the network of Sentinel General Practitioners. Arch Publ Health 2010; 68(2): 62-7

12. Spackman E, Senne DA, Myers TJ, Bulaga LL, Garber LP, Perdue ML et al. Development of a RealTime Reverse Transcriptase PCR Assay for Type A Influenza Virus and the Avian H5 and H7 Hemagglutinin Subtypes. J Clin Microbiol 2002, 40: 3256-60

13. Van Elden LJR, Nijhuis $P$, Schipper R, Schuurman R, Van Loon AM. Simultaneous Detection of Influenza Viruses A and B Using Real-Time Quantitative PCR. J Clin Microbiol 2001, 39: 196-200

14. Centers for Disease Control and Prevention. CDC protocol of realtime RTPCR for influenza $A(H 1 N 1) .6$ October 2009. Available from: http://www.who.int/csr/resources/publications/swineflu/CDCRealtimeRTPCR_SwineH1Assay-2009_20090430.pdf 